\title{
Geometrical correction and photogrammetric approach in thermographic inspection of buildings
}

\author{
by P. Bison*, A. Bortolin*, G. Cadelano*, G. Ferrarini*, K. Furlan*, E. Grinzato*
}

^ITC-CNR., Corso Stati Uniti 4, 35127 Padova Italy, gianluca.cadelano@itc.cnr.it

\begin{abstract}
Thermography is now commonly used as a tool for the qualitative inspection of buildings, for different purposes such as energy monitoring, moisture mapping and many others. All these controls need to correctly locate in space the different phenomena observed and evaluate the area affected by the phenomena. Furthermore, when thermography is applied quantitatively and fluxes are evaluated, the precise area affected by the process is of paramount importance. This paper intends to approach the issue to obtain a correct three-dimensional reconstruction of the building that will promptly integrate all the information to the surface, such as wall temperature, optical absorption coefficient, etc.

For this purpose, at first the influence of the visual and IR optics calibration is addressed. After that, sets of images taken in the visible and infrared spectrum are processed for the geometric correction, mosaicing and 3D reconstruction. The ultimate output is a $3 \mathrm{D}$ model of the object implemented with matching sets of data (e.g. temperature, heat flux, etc.) allowing an easy visualization and their correct positioning in space. The main result of this process is the capability of quantitative analysis of thermal processes acting on the building.
\end{abstract}

\section{Introduction}

Thermographic applications in the inspection of buildings such as the evaluation of energy performance, the indoor comfort or the monitoring of the water vapor exchanged between historical painted surfaces subject to degradation and the surrounding air, are increasingly frequent and of well-proven reliability[1]. However, the quality of the inspection results obtained depends by the necessity to integrate them with information about their location in the geometry of the building and to metrically quantify the dimension of the observed phenomena[2].

The geometrical model of the building can be based on existing drawings or direct measurements, even if nowadays the automatic scanning using a laser range unit is becoming more and more popular and effective. Another suitable method is photogrammetry[3].

Even the best thermographic equipment now available in commerce for civil uses is unfortunately not appropriate to apply standard photogrammetric procedures. Therefore, it is necessary not only to apply an optical calibration to thermographic cameras and lenses[4], but also to use complementary information from other instruments. In particular, the thermographic device should be integrated with a photocamera to allow high-resolution geometric information and, combined with computer algorithms, to compensate for the weaknesses of thermal imaging systems, increasing at the same time the information content for the benefit of a more complete inspection[5].

\section{Methods}

The methodology involves the use of standard hardware components and commercially available parts. The devices used for the experimental tests are a thermographic camera and a visible camera. The goal is not to obtain extreme geometrical accuracy but a simplified model, that should be compatible with the degree of precision required for the thermal inspection (centimetric resolution in most cases).

Regarding image processing procedures, for both IR and visible spectrum data, commercial software are used, but applying ad hoc procedures. In particular PhotoModeler $\odot$ and Matlab $®$ software allow to obtain metrically correct and point-to-point matched textures and to combine surface data distributions.

Images taken with digital camera are used mainly to create the 3D model of the building analyzed. The same data can be useful as photographic textures and to evaluate parameters such as absorption coefficient, shadows evaluation and other quantities depending on the equipment used (e.g. multispectral analysis, near IR, etc.)[6].

Thermal images, after being corrected by the effects of aberration due to the optical, are straightened and processed with algorithms, similar to those used in photographic stitching and panorama software, specifically developed to be used with thermograms, in order to maintain radiometric information but increasing pixel resolution. From these radiometric data, using special techniques, it is possible to gain additional information such as maps of distribution of moisture, temperature and air velocity, heat flow [7].

The main advantage of such an approach is the easy and accurate (1 cm accuracy) point-to-point matching, for each of the analyzed surface, of all the parameters obtained from the different analysis. 
The final result of this procedure is an interactive, multilayer, three-dimensional model where each pixel has several associated values (temperature, heat flux, absorption coefficient, etc). The end result (3D model and data) is adaptable to different applications, e.g. exploiting simulated temperatures computed by Finite Elements Method (FEM).

Finally, results are exported to Google Earth ${ }^{\circledR}$ for georeferencing, shadow projection forecasting and other uses.

\section{Camera calibration}

The camera calibration has a key role in the process of restitution of geometrical thermographic surveys. In particular, it must be emphasized that the state of the art at the international level of this operation, as regards the thermographic systems, is currently very limited, but constitutes a field of growing interest. The cameras are usually calibrated only by the radiometric point of view, in order to provide accurate values of temperature, but not from the geometrical point of view. In this chapter the calibration of FLIR Systems SC660 model is presented, whose main characteristics are presented as follows:

Table 1. Main specifications of FLIR Systems SC660 thermographic camera with built in photographic camera

\section{FLIR Systems SC660 specifications}

\begin{tabular}{ll}
\hline IR image resolution & $640 \times 480$ pixel \\
\hline Spatial resolution & $0.65 \mathrm{mrad}\left(24^{\circ}\right)$ \\
\hline Spectral range & $7.5-13 \mu \mathrm{m}$ \\
\hline Image frequency & $30 \mathrm{~Hz}$ or $60 / 120 \mathrm{~Hz}$ windowed \\
\hline Focus & Automatic or Manual \\
\hline Temperature range & From $-40{ }^{\circ} \mathrm{C}$ to $+1500{ }^{\circ} \mathrm{C}$ \\
\hline Thermal sensitivity & $30 \mathrm{mK}$ at $30{ }^{\circ} \mathrm{C}$ \\
\hline Thermal accuracy & $\pm 1{ }^{\circ} \mathrm{C} \mathrm{or} \pm 1 \% / \pm 2{ }^{\circ} \mathrm{C} \mathrm{or} \pm 2 \%$ \\
\hline
\end{tabular}

The analytical calibration of photocameras, including the integrated one into the thermalcamera and nonprofessional models, was instead the subject of careful scientific research over the years, which led to the development of different methods and mathematical models. They are divided into two categories, respectively named "on - the - job calibration" and "self - calibration", which are both based on the use of a bundle-adjustment algorithm [4] with the following variables:

$(\mathrm{XO}, \mathrm{YO}, \mathrm{ZO})=$ coordinates of the center of projection;

$(\Omega, \varphi, k)=$ the angular structure of the chamber in the external system;

$\mathrm{c}=$ distance of the main camera;

$\left(X_{P}, Y_{P}\right)=$ coordinates of the principal point, which is the intersection of the optical axis with the image plane;

$\left(\mathrm{K}_{1}, \mathrm{~K}_{2}, \mathrm{~K}_{3}\right)=$ symmetrical radial distortion coefficients

$\left(\mathrm{P}_{1}, \mathrm{P}_{2}\right)=$ asymmetric and tangential distortion coefficients of radial distortion, which are due to the decentralization of the lenses to the optical axis, but generate negligible effects compared to the radial distortion coefficients symmetrical and, therefore, are not generally considered in the calibration procedures.

The most common types of distortion are known as "cushion", if the distorted image has concave curvature, or "barrel", if the curvature appears convex. Therefore, particular importance is the use of the calibration procedure that allows to determine the parameters described above and to intervene to reduce the incidence of such errors in the use of frames, using the following equations:

$d r=K_{1} r^{2}+K_{2} r^{4}+K_{3} r^{6}+\ldots$
$d t x=P_{1}\left(r^{2}+2 x^{2}\right)+2 P_{2} x y$
$d t y=P_{2}\left(r^{2}+2 y^{2}\right)+2 P_{1} x y$

in which:

$r$ is the radius of the lens;

$\mathrm{dr}$ is the symmetrical radial distortion of the lens;

$\mathrm{dtx}$ is the asymmetric radial and tangential distortion of the lens along the $\mathrm{x}$ axis; $\mathrm{dty}$ is the asymmetric radial and tangential distortion of the lens along the y axis; $x$ and $y$ are the distances between the lenses. 
Calibration "on - the - job" provides the determination of parameters of interest in a process closely related to the photogrammetric survey, from frames depicting the object of study and from points of known coordinates. The execution context of these operations may, however, cause problems in the calibration phase, due to conditions that may occur during the survey, such as specific location choices or inadequate intake patterns.

Unlike this approach, in "self - calibration" the determination of the parameters is carried out separately from the inspection phase, using calibration grids specially made that are known with good precision the coordinates of the points that constitute them. For calibrations performed in this paper the authors decided to use the grid provided with the software PhotoModeler ${ }^{\odot} 2011$, consisting of 100 points, four of which are arranged near the corners and are encoded to be recognizable within each frame.

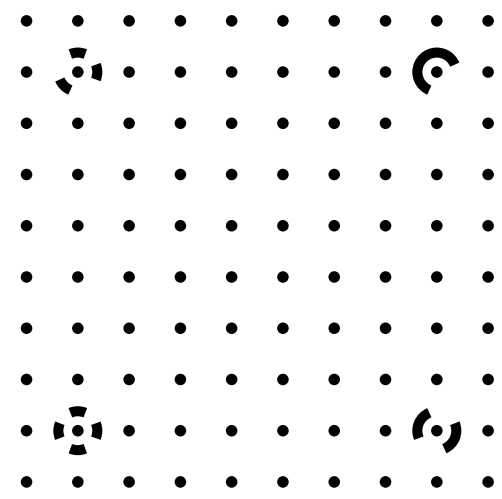

Fig. 1. PhotoModeler@ 2011 standard calibration pattern, consisting of 100 regularly disposed dots and four control markers.

The effectiveness of the calibration depends on the quality of scanned images: a major source of error is due to the presence of the "zoom" function in compact cameras. If the camera is not a fixed lens type it is necessary to make sure not to use this feature when shooting in so far as it goes change the focal length of the lens. The accuracy of the method can be increased also by acquiring, in addition to central prospective shots, images obtained by rotating the camera $90^{\circ}$ clockwise and counterclockwise, in order to avoid correlation between the orientation parameters external and internal. Finally, the image area should be covered as much as possible with the calibration grid: some frames may not see fulfilled this condition, but overall, taking all 12 images, the image area must be at least $80 \%$ covered by the grid.

Calibration procedures were performed following two different approaches. The first involves the use of a free toolbox in Matlab® [8] while the second makes use of commercial software PhotoModeler` 2011. The latter was also used for the modeling of three-dimensional building elements, then exported, making them available for further processing and studies using the software Google Earth@ and Google Sketch Up@).

In the examples provided at [8] the calibration grid shown in figure 2 is used, in which points of interest are given by the vertices of the squares of a chessboard pattern.

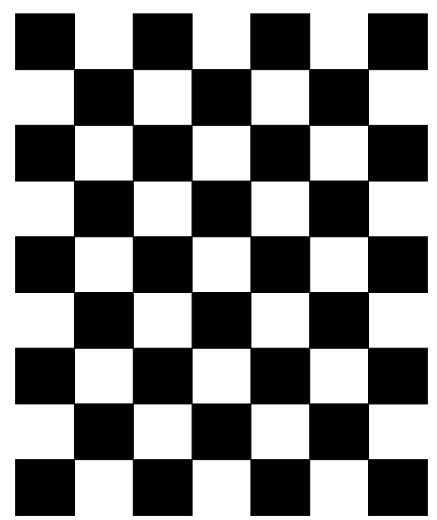

Fig. 2. Camera Calibration Toolbox for $M A T L A B \Theta$ by Caltech standard calibration pattern, consisting of a $7 \times 9$ black and white chessboard.

Laboratory tests were performed with both grids using an entry level Casio Exilim EX-Z11 camera and the embedded photo camera of FLIR Systems SC660 thermal camera. The main source of error in this procedure is that the geometrical features of the grid are set manually by the user, which implies a limited accuracy. 
The actions described up to this point for a camera calibration in the visible may be repeated, with appropriate devices, even for equipment operating in the infrared range, such as the thermal sensor of FLIR Systems SC660. The state of the art on this subject is, as already mentioned, is quite primitive. However, the calibration of a thermal camera is not trivial, because it is necessary to produce a pattern that is sufficiently contrasted when it is captured in thermal IR range. There are different approaches to the problem [2]; both the thermal and the radiometric method has been explored. Using the radiometric method the images are no longer obtained by temperature variations of the pattern feature, but by the difference in the emissivity $\varepsilon$ of the surface. The grid is constituted by a perforated aluminum thick plate, thus allowing the vision of a $10 \times 10$ matrix of points, such as that provided by standard Photomodeler $($ ) template. One issue is the creation of the four corner points encoded. Excluding the possibility to affect the plate, it was decided to proceed placing black cardboard at the four vertices reproducing the encoded points due to difference of emissivity between cardboard and aluminum.

After the calibration procedure only 96 points were correctly recognized, while for the other four (encoded) have been confused with background, causing an early termination by the software during the initialization process. In order to have a much more accurate grid a sheet of acetate paper, on which was printed a scaled version of the same pattern, was placed on the aluminum plate, caring of matching the black dots with the holes below. The points recognition by the camera was achieved by turning on a spotlight for a few moments before each image acquisition. The operating principle of the method relies on the fact that the light emitted by the lamp can heat the black markers much more than the reflective background. However, the calibration program has not been able to recognize a number of points sufficient to allow starting the calibration procedure. This is due to the fact that the spotlight, while remaining switched on for a few moments, causes a heating of the markers which spreads on the surface that lowers the pattern definition.
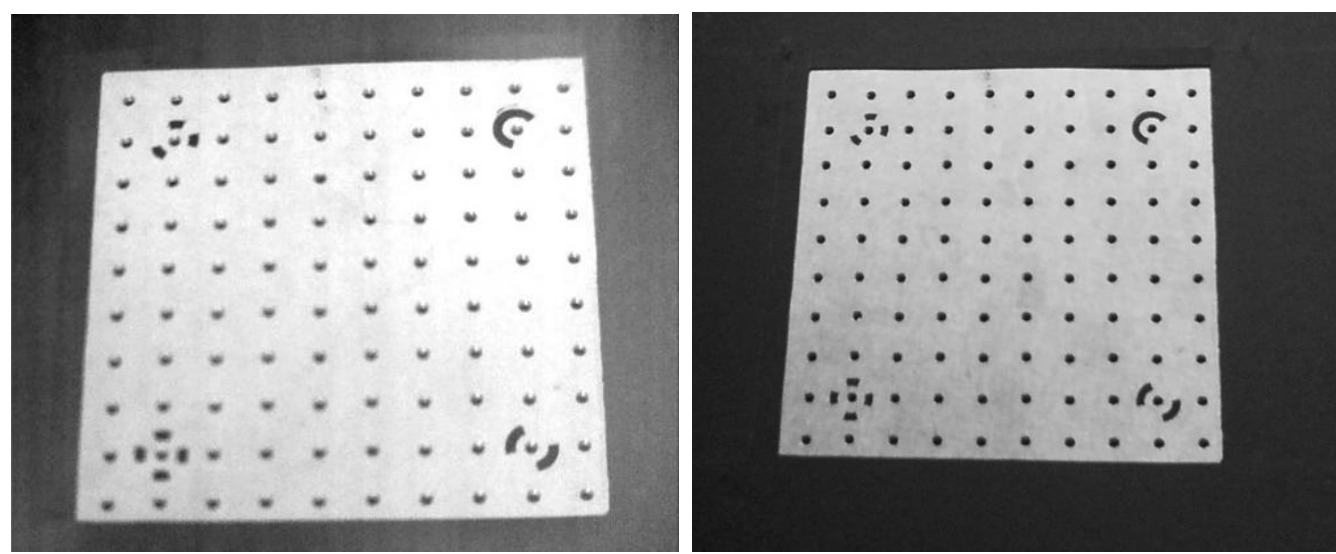

Fig. 3. Calibration patterns used for IR camera calibration: on the left holes are drilled on the aluminum surface, on the right an acetate sheet is printed with black ink.

The calibration process was finally achieved replacing the spotlight with two flash lamps of considerable power $(4.8 \mathrm{KJ}$ in $5 \mathrm{~ms})$ and minimizing the delay between heating and image acquisition, in order to limit the impact of thermal diffusion. Since the process is very fast, this procedure must be performed with high frequency - acquisition cameras, such as $30 \mathrm{~Hz}$ for the FLIR Systems SC660 thermal camera.

This procedure was followed by the calibration process that recognized a suitable number of points, including special encoded points, obtaining good results: the Point Marking Residuals Maximum was equal to 0383 pixels ( $<1$ pixel) and the Point Marking Residuals Maximum RMS was equal to 0205 pixels ( $<0.5$ pixels).

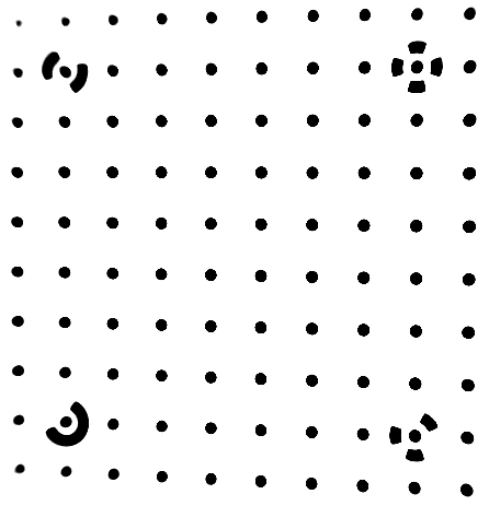

Fig. 4. Binarized thermal image, reproducing the PhotoModeler@ 2011 standard calibration pattern after the release of two flash bulbs, recorded with FLIR Systems SC660. 
This IR camera has also a built-in visible camera, with a resolution equal to 3.2 megapixels. For the calibration of this device the results that were obtained in this way were not acceptable: the Point Marking Residuals Maximum was equal to 1,247 pixels (> 1 pixel) and the Point Marking Residuals Maximum RMS was equal to 0,694 pixels (> 0.5 pixels).

\section{Photo stitching, IRstitching and 3D models}

The straightening process consists in the elaboration of an image of a planar object that allows the transformation from central projection (perspective) to orthogonal projection. This is a photogrammetric procedure that allows to correct the perspective effect due to the non-orthogonality of the shot from a single image through the process of orientation and restitution. In particular, the restitution process consists in straightening the entire image using a software that allows a digital resampling. The image relative to the plane object that is obtained is no perspective or distortion affected and, therefore, provides a correct measure of the distances.

An Orthophoto consists in producing an image of a non-planar object that allows the transformation from central projection (perspective) to orthogonal projection. This is also a photogrammetric procedure, which again works on a single image, that can be applied only if a model of the surface $S=f(X, Y, Z)$ of the detected object is known. The Digital Surface Model (DSM) of the surface can be built from known coordinates points or with other detection techniques.

In both cases the resulting image can be stored in raster format (e.g. .bmp, .png), or may be subjected to manual or automatic vectorization and, therefore, may be stored in vector format (e.g. .dxf, .dwg). The straightening process provides best results depending on the quality of the image, while the orthophoto is more affected by the quality of the geometrical information. One of the main goals of this work consists in reconstructing, with a good level of accuracy, a 3D model of a building from images taken in the visible and thermal IR wavelengths.

Following the calibration procedures, it is possible to proceed with the description of the creation of threedimensional models. A small building was selected as test case: several images were taken with Casio Exilim EX-Z11 and processed with PhotoModeler@ as shown in figure 5.

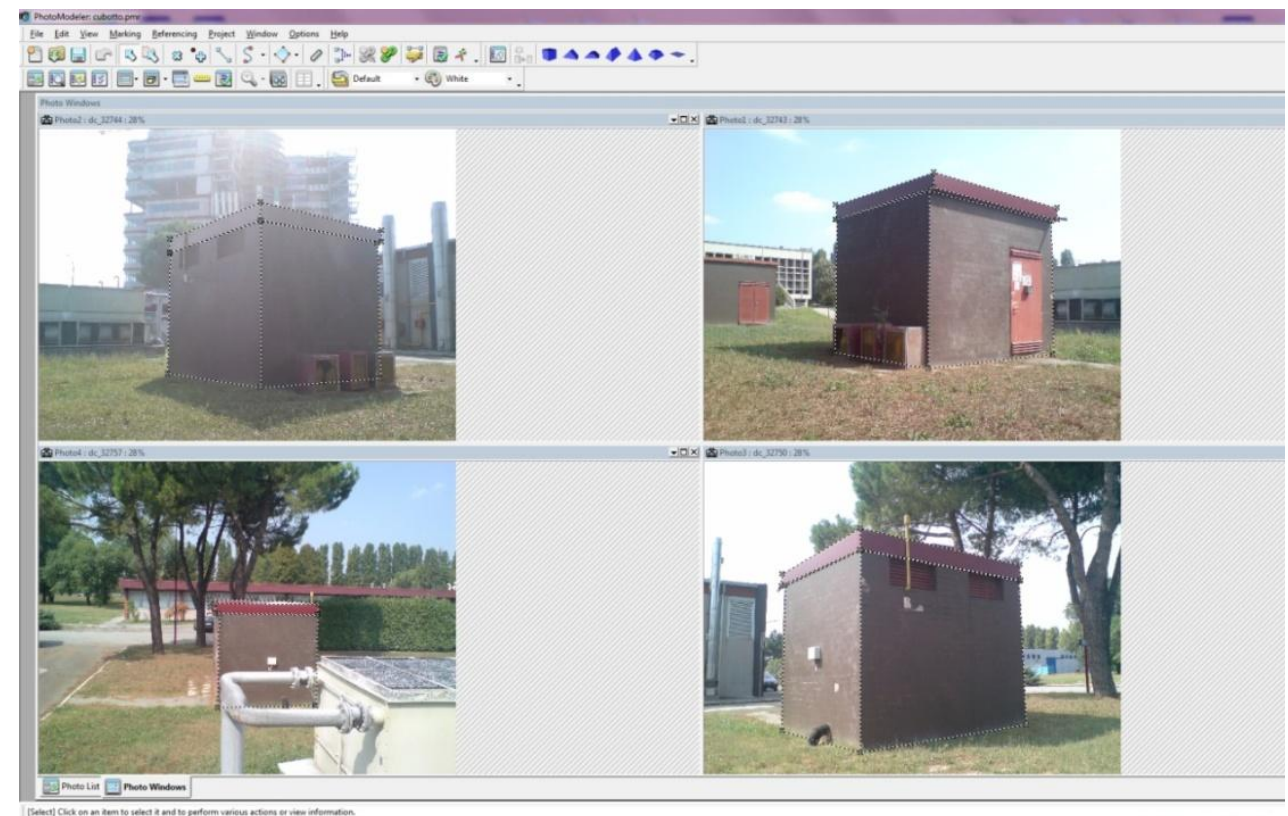

Fig. 5. Four photographic images of a simple building are used to create a three dimensional model using PhotoModeler(.

Four photographic images were used to create a three dimensional model of the building. To orient the images is necessary to draw on each image lines in order to identify horizontal and vertical edges of the building and to establish links between homologous points on different images. The next step is to assign features to the surfaces forming the solid obtained by associating a specific set of textures derived from the original images or in general including the temperature distribution. In this case four photographic images were applied as textures to the virtual surfaces of the model as shown in figure 6. 

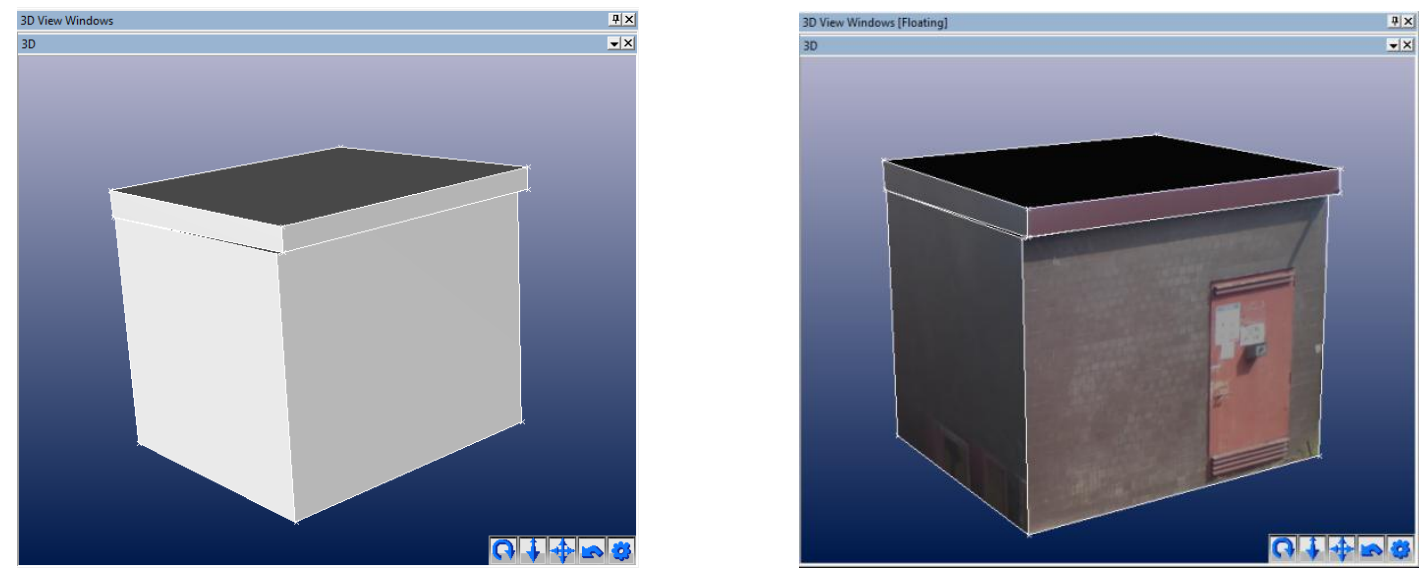

Fig. 6. On the left the finished $3 D$ model of the building, before the application of textures as shown on the picture on the right.

The same procedures used for the creation of a 3D model from photographic images can be applied to create a project based on thermograms recorded in the infrared range, visible in figure 7.

However, it must be pointed out that by following this procedure the resulting model denotes much less accuracy than the 3D model generated starting from photographic images. This is largely due the uncertainty in the identification of the edges of the building that is a consequence of the low spatial resolution of the thermographic camera, even if the FLIR SC 660 is commonly considered top level for this feature.

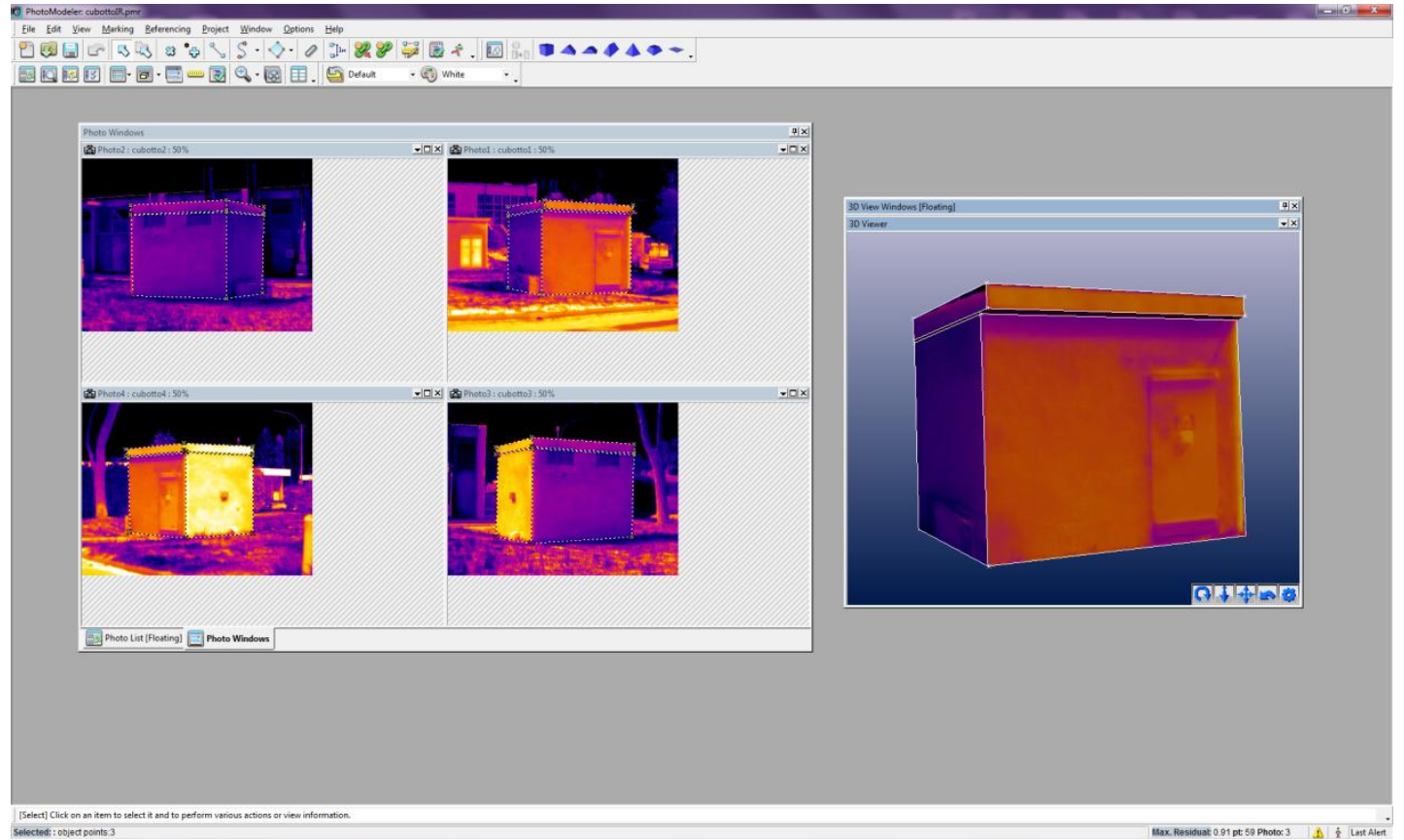

Fig. 7. Four thermal images of a simple building are used to create a three dimensional model using PhotoModeler(C.

In table 2 are presented the results obtained measuring the surfaces of the test building with different devices. The area values of the surfaces of the three-dimensional models obtained through the use of thermal images and photographs, taken with calibrated and uncalibrated thermographic and photographic cameras, have been compared with the direct measurement of the building. 
Table 2. 3D model areas comparison. Different results are obtained depending on the camera type (thermographic or photographic) and the calibration process.

SW-SE NW-SW SE-NE NW-SE

\begin{tabular}{|c|c|c|c|c|c|}
\hline Camera model & Calibration & Surface $\left(m^{2}\right)$ & Surface $\left(\mathrm{m}^{2}\right)$ & Surface $\left(\mathrm{m}^{2}\right)$ & Surface $\left(\mathbf{m}^{2}\right)$ \\
\hline $\begin{array}{l}\text { (1) Thermographic camera } \\
\text { FLIR SC660 (1) }\end{array}$ & No & 10.75 & 9.87 & 9.58 & 10.75 \\
\hline $\begin{array}{l}\text { (2) Thermographic camera } \\
\text { FLIR SC660 }\end{array}$ & max residual 0.115 & 10.85 & 8.94 & 9.20 & 11.47 \\
\hline (3) FLIR SC660 Built-in camera & No & 10.42 & 8.12 & 8.74 & 10.65 \\
\hline (4) FLIR SC660 Built-in camera & max residual 1.951 & 10.44 & 8.42 & 8.68 & 10.14 \\
\hline (5) FLIR SC660 Built-in camera & max residual 0.657 & 10.40 & 8.33 & 8.58 & 9.95 \\
\hline (6) Casio Exilim EX - Z11 & max residual 0.786 & 10.44 & 8.52 & 8.94 & 10.38 \\
\hline & Surface $($ actual $)=$ & 10.40 & 8.55 & 8.55 & 10.40 \\
\hline
\end{tabular}

This analysis of the results is limited to the comparison of the extention of the surfaces, and does not take into account the arrangement and orientation in space of 3D models. The lack of such a info into the graph of figure.8 explain the apparent contraddiction between the camera 4 and 5.

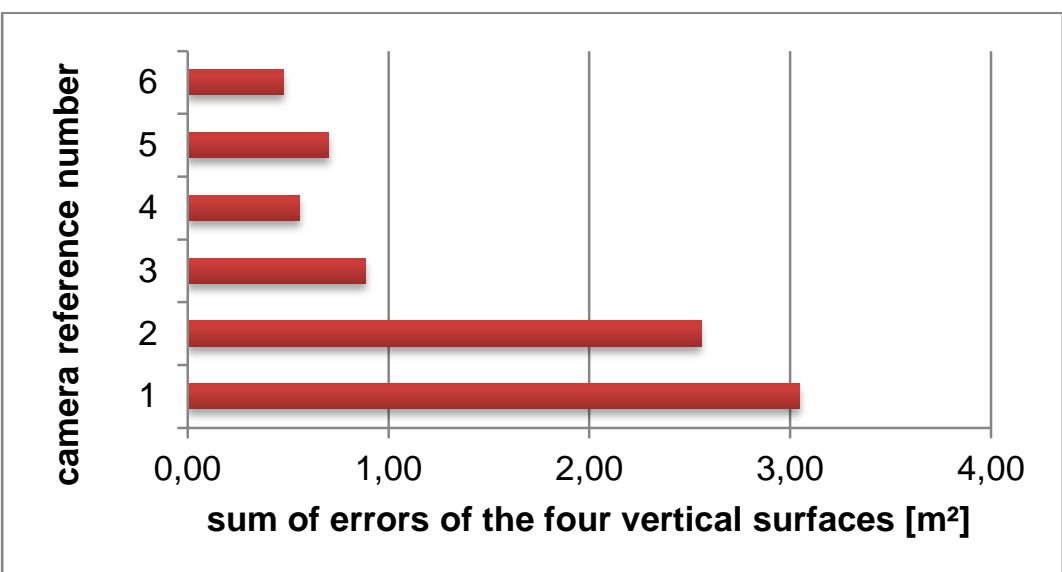

Fig. 8. Comparison between six 3D models of the same building made with different cameras, based on the sum of errors of the four vertical surfaces.

After defining for each point a set of geomatic features as latitude, longitude and altitude, 3D reconstruction can also be imported into Google Earth $\AA$, as shown in figure 9 . This geotagging process is important, not only because the building can be viewed within a local context, but also because in this way is possible to known the provisions of the surfaces over to cardinal directions. Therefore, shading effects of lateral objects may be easily studied at any time. 


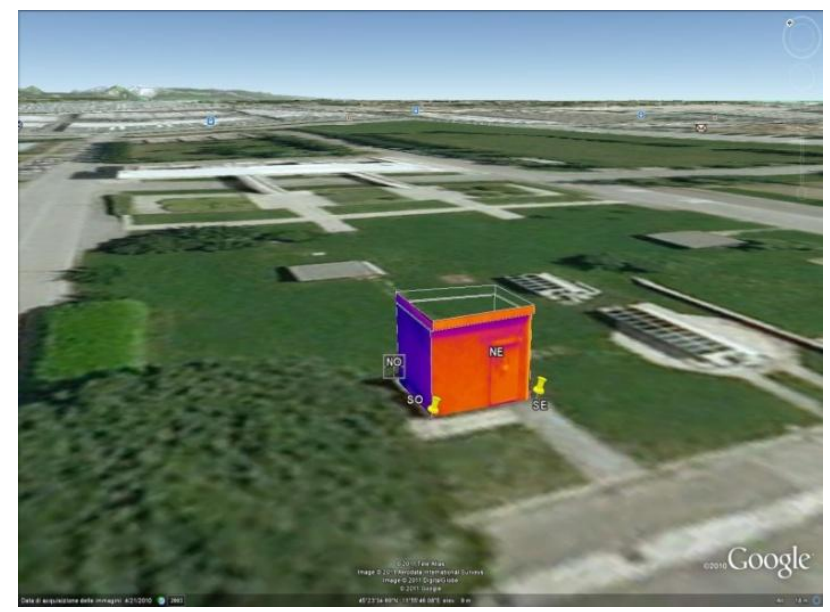

Fig. 9. Three dimensional model of a simple building, based on thermal images, geotagged in Google Earth $\AA$.

In order to improve the achieved results an alternative methodology, which implies the integrated use of different software, is now ongoing. The three-dimensional reconstruction of a building is performed, using a software as PhotoModeler(c), from images in the visible wavelengths, but the acquisition is aided with markers. Unlike the case previously described, acquired thermograms are processed through ad hoc scripts developed in Matlabß. Alternatively, the model can be drawn directly using various commercial software. The application of textures is performed using Matlab $\AA$, which is able to process data acquired from different sensors (i.e. photographic camera and thermal camera) and position them accurately in the modeled geometry of the building. Key of success in this technique is the implementation of special markers that can be automatically recognized in both visible and thermal images. The peculiarity of this method is, therefore, the ability to integrate the various images captured by developing a strategy to preserve their geometric and radiometric characteristics.

Another important feature of this new way (named alRview) to inspect interiors, especially of wide surfaces, is the implementation of the procedure in an automatized system consisting of a robotized pan-tilt unit on which are fixed a camera and an industrial grade infrared camera, now in prototypal stage of development. The images acquired by a thermographic camera installed on the pan-tilt unit are automatically processed. The system, visible in figure 10, relies on a special grid made with visible/IR markers that is put near the wall under inspection.

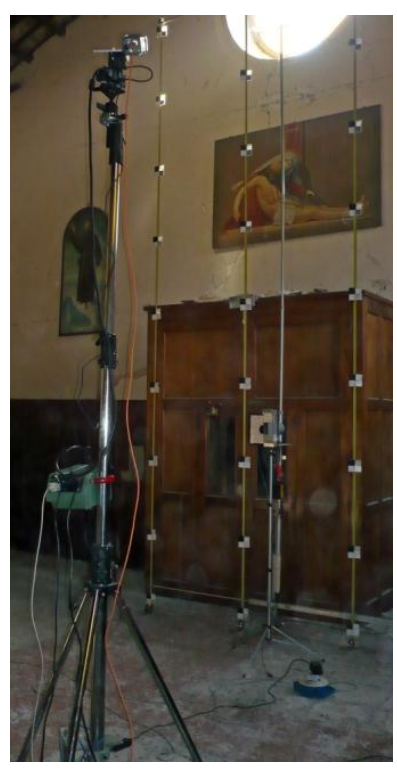

Fig. 10. alRview system inside an historical church.

This system was recently used in the survey of San Vito alla Rivera church in L'Aquila, Italy (March 2012). Figure 11 represents some of the results obtained soon after the end of the survey. 440 individual thermograms were acquired in less than 30 minutes, producing a full coverage area of about $250 \mathrm{~m}^{2}$ at 1 pixel=1 $\mathrm{cm}^{2}$ resolution and accuracy. 

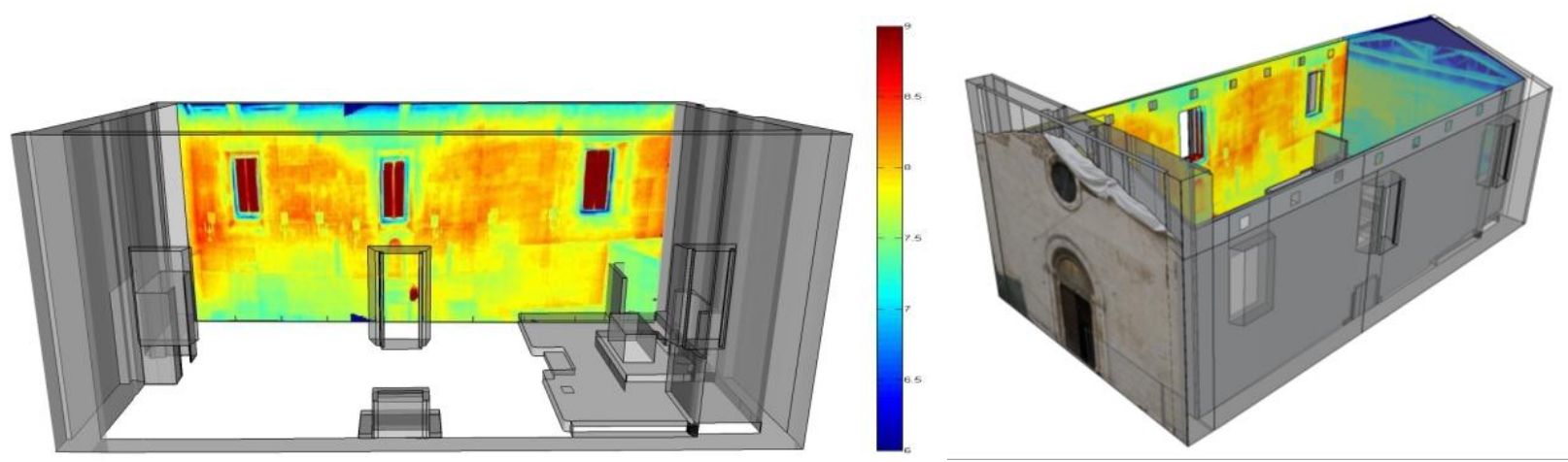

Fig. 11. Two views of a simplified $3 D$ model of San Vito alla Rivera church where some of the result obtained by alRview system are applied as textures (i.e. wall surface temperature).

\section{Conclusions}

This study has identified and developed a method that allows the use of thermography not only as a radiometric quantitative and geometrically qualitative tool in the analysis of structures, but also as a technique for quantitative measurement of the thermal processes that occur in space. It is fundamental the geometrically correct reconstruction of three-dimensional models of buildings, that allows to locate and accurately evaluate the geometric extension of phenomena and the gradients of temperature. The geometrical calibration of different image acquisition devices is necessary, especially for thermographic cameras, in order to compensate for lens distortion effects.

The commercial systems currently offered are inadequate to solve this problem. Basically, two kinds of survey are available: the first one implies qualitative consideration about surface evaluation even despite an high level radiometric evaluation; the second one make use of advanced photogrammetric methods or other powerful techniques such as laser scanner, but eventually lacks of efficiency due to high costs and slow survey speed. The velocity problem is not trivial, because the thermal process is dynamic and it is incorrect to compare data recorded on different conditions. Indeed, this is important as other aspects like the accuracy of the temperature measurements. In fact, the survey time has to be compatible with the speed of the phenomena observed.

The new system alRview is now under development, with the mission to cover these drawbacks making intense implementation of automatized procedures through the robotization of acquisition devices and the implementation of artificial vision algorithms. Furthermore, it will record not only the surface temperature, but also the thermodynamic conditions of the air, on local basis.

\section{REFERENCES}

[1] Grinzato, E., Vavilov, V., Kauppinen, T., 1998. "Quantitative infrared thermography in buildings". Energy and Buildings, Vol. 29, Issue 1, pp. 1-9.

[2] Lagüela, S., Martínez, J., Armesto, J., Arias, P., 2011. "Energy efficiency studies through 3D laser scanning and thermographic technologies". Energy and Buildings, Vol. 43, pp. 1216-1221.

[3] Cabrelles, M., Galcerà, S., Navarro, Lerma, J. L., Akasheh, T., Haddad, N., Integration of 3D laser scanning, photogrammetry and thermography to record. Architectural monuments. $22^{\text {nd }}$ CIPA Symposium, October 11-15, 2009, Kyoto, Japan.

[4] Remondino, F., Fraser, C., 2006. "Digital camera calibration methods: considerations and comparisons". International Archives of Photogrammetry, Remote Sensing and Spatial Information Sciences, Vol. XXXVI.

[5] Barazzetti, L., Scaioni, M., Remondino, F., 2010. "Orientation and 3D modelling from markerless terrestrial images: combining accuracy with automation”. The Photogrammetric Record, Vol. 25, Issue 132, pp. 356-381.

[6] Grinzato E., Bison P., Girotto M., Volinia M., "Sull'intonaco e oltre: diagnostica non distruttiva per I monitoraggio del patrimonio storico-monumentale misura in situ dell'effusività termica". Atti del "13 Congresso AIPnd", Roma, 15-17 ottobre 2009, http://www.ndt.net (in Italian).

[7] Grinzato, E., Bison, P., Cadelano, G., Peron, E., 2010. "R-Value estimation by local thermographic analysis". Thermosense XXXII, Vol. 7661, 76610H-2.

[8] Camera calibration toolbox for Matlab ${ }^{\circledR}$, developed by J.-Y. Bouguet http://www.vision.caltech.edu/bouguetj 